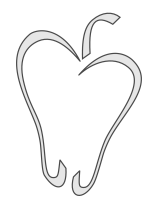

ISSN $1852-4249$

\title{
Reply to the Commentary on "Critical phenomena in the spreading of opinion consensus and disagreement"
}

\author{
A. Chacoma ${ }^{1}$ D. H. Zanette ${ }^{1,2 *}$
}

1. Taking into account the reviewer's concern (see Ref. [1]), we have replaced the right panel of Fig. 1 in our main article (see Ref. [2]) by a plot showing the evolution of an entire 200-agent array. We hope that this dissipates the possible confusion pointed out by the reviewer. The caption and the main text have been modified accordingly.

2 . Indeed, the equivalence between the onedimensional (1D) versions of the voter model with nearest-neighbor interactions and of diffusionlimited binary annihilation $(A+A \rightarrow 0)$ has been recognized since the first studies of coarsening processes [3]. The boundaries separating domains with different opinions in the 1D voter model move as random walkers, which annihilate with each other when they meet during their motion. In view that, in the case of a linear array with $p_{D}=p_{C}=1$, our model reduces to two mutually intercalated voter systems, the scaling laws of binary annihilation also apply to our results. It is well known, for instance, that the number of particles $a(t)$ in $1 \mathrm{D}$ diffusionlimited annihilation decays with time as $a \sim t^{-1 / 2}$ [4]. This implies that, in a finite system, the time needed for complete annihilation of an initial (even) number of particles, $a(0)$, goes as $T \sim a(0)^{2}$. In our

\footnotetext{
*E-mail: zanette@cab.cnea.gov.ar

1 Instituto Balseiro and Centro Atómico Bariloche, 8400 San Carlos de Bariloche, Río Negro, Argentina.

2 Consejo Nacional de Investigaciones Científicas y Técnicas, Argentina.
}

model, in turn, the initial number of boundaries between opinion domains can be seen to behave as $b(0) \sim n_{+}(0) n_{-}(0) N$, where $n_{ \pm}(0)$ is the initial fraction of agents with each opinion, and $N$ is the system size. The above result indicates that the time needed for all the boundaries to disappear is $T \sim n_{+}^{2}(0) n_{-}^{2}(0) N^{2}$. In other words, as illustrated by the results shown in the lower panel of Fig. 2, the product $N^{-2} T$ depends only on the initial concentration of each opinion. A similar argument makes it possible to show that the probability of reaching consensus $P_{\text {cons }}$ depends on $n_{ \pm}(0)$, but it is independent of the system size, as shown in the upper panel of the same figure.

On the other hand, the possible connection between the case $p_{D}, p_{C} \neq 1$ and branchingannihilation random walks, is less clear. As remarked by the reviewer, this connection should, in turn, establish a link with the universality class of directed percolation. However, the facts that our model exhibits multiple absorbing states and that there is no phase where fluctuations persist at asymptotically long times (as well as the absence -in the 1D case, where the connection is expected to hold - of nontrivial critical exponents) do not seem to suggest a relation to that universality class [5]. The point, nevertheless, is worth considering in future work.

3. Certainly, as acknowledged in the paper's final section, the most important direction in which our model should be extended is to consider more 
Papers in Physics, vol. 6, ART. 060005 (2014) / A. Chacoma et al.

complex topologies, in particular, those that represent real-life social systems. The one- and twodimensional arrays studied in the paper are just a convenient — and probably the simplest - way of defining the groups of agents that participate in the opinion dynamics. It must be realized, however, that the existence of an underlying network of social contacts (either ordered or not) is not necessary to specify the structure of groups relevant to our class of models. In fact, the most general definition of the group structure is to provide a list of all the groups present in the population, enumerating the agents that belong to each group. This procedure encompasses all the possible partitions into groups of any given population - even those that cannot be represented by means of an underlying network [6] - and thus allows for the consideration of any degree of complexity compatible with the population size. The active group $G$ and the reference group $G^{\prime}$ involved in each interaction event can then be chosen - for instance, at randomfrom the list that specifies the group structure.

Note that, from this perspective, a network whose topology is entirely defined by the list of all its links - is nothing but a structure formed by a set of two-agent groups. In this sense, the notion of group structure generalizes that of network, introducing a kind of higher-degree connection between population members $[6,7]$.
[1] F Bagnoli, Commentary on "Critical phenomena in the spreading of opinion consensus and disagreement", Pap. Phys. 6, 060004 (2014).

[2] A Chacoma, D H Zanette, Critical phenomena in the spreading of opinion consensus and disagreement, Pap. Phys. 6, 060003 (2014).

[3] S Redner, A guide to first-passage processes, Cambridge University Press, Cambridge (2001).

[4] A S Mikhailov, A Y Loskutov, Foundations of synergetics II. Chaos and noise, Springer, Berlin (1996).

[5] H Hinrichsen, Nonequilibrium critical phenomena and phase transitions into absorbing states, Adv.Phys. 49, 815(2000).

[6] D H Zanette, Beyond networks: Opinion formation in triplet-based populations, Phil. Trans. R. Soc. A 367, 3311 (2009).

[7] D H Zanette, A note on the consensus time of mean-field majority-rule dynamics, Pap. Phys. 1, 010002 (2009). 